\title{
Efeito do intervalo entre recrutamentos foliculares na superovulação de vacas da raça Holandesa não-lactantes
}

[Effect of interval between follicular recruitments on superovulatory response in non-lactanting Holstein cows]

\author{
R.M. Santos ${ }^{1,3}$, J.L.M. Vasconcelos ${ }^{2 *}$ \\ ${ }^{1}$ Aluna de pós-graduação - FCAV-UNESP - Jaboticabal, SP \\ ${ }^{2}$ Faculdade de Medicina Veterinária e Zootecnia - UNESP \\ Caixa Postal 560 \\ 18618-000 - Botucatu, SP \\ ${ }^{3}$ Bolsista da FAPESP
}

\begin{abstract}
RESUMO
Vacas da raça Holandesa não-lactantes, distribuídas em dois grupos, foram sincronizadas com o protocolo Ovsynch modificado. No dia sete (dia $0=$ dia do segundo GnRH), o grupo 7 (G-7; n=19) recebeu CIDR usado previamente por cinco dias e 100mcg de GnRH, e o grupo 14 (G-14; n=21), CIDR e 25mg de PGF2 $\alpha$. No dia 14 foi aspirado o folículo dominante (FD), trocado o CIDR usado por um novo e foram aplicados 25mg de PGF2 $\alpha$. Iniciou-se o tratamento com FSH 36h depois, removeu-se o CIDR com o sétimo FSH e aplicou-se GnRH 36h depois. As inseminações foram feitas 12 e $24 \mathrm{~h}$ depois. Recuperaramse os embriões sete dias depois da inseminação artificial. O diâmetro do FD no G-7 foi 13,1 $\pm 0,57 \mathrm{~mm}$ no dia sete e $11,2 \pm 0,57 \mathrm{~mm}$ no dia 14 . O diâmetro FD persistente no G-14 aumentou de $12,6 \pm 0,55 \mathrm{~mm}$ no dia sete para $16,4 \pm 0,55 \mathrm{~mm}$ no dia $14(\mathrm{P}<0,001)$. O número de folículos $\geq 8 \mathrm{~mm}, 48 \mathrm{~h}$ após o início do tratamento com FSH, foi maior $(\mathrm{P}<0,05)$ no G-7 $(15,6 \pm 0,05)$ que no G-14 $(12,5 \pm 0,05)$. Não foi detectado efeito de tratamento sobre o número de corpos lúteos e de embriões. $\mathrm{O}$ menor intervalo entre recrutamentos foliculares aumentou o número de folículos recrutados, porém não alterou a quantidade e a qualidade dos embriões produzidos.
\end{abstract}

Palavras-chave: bovino, aspiração folicular, embrião, FSH, superovulação

\begin{abstract}
Dry Holstein cows were synchronized by a modified Ovsynch protocol. On day 7 (day $0=$ day of the second GnRH), group $7(G-7 ; n=19)$ received an used CIDR and GnRH (100 $\mu$ g). Group $14(G-14 ; n=21)$ received an used CIDR and PGF2 $\alpha$ (25mg). On day 14, the dominant follicle (DF) was removed, by aspiration; the used CIDR was changed for a new CIDR plus PGF2 $\alpha$. Thirty-six hours later, the FSH treatment was initiated. On the seventh injection of FSH, the CIDR was removed. Thirty-six hours after, the cows received GnRH. Donors were inseminated 12 and 24 h after the GnRH injection. Embryos were recovered seven days after AI. The DF diameter in $G-7$ was $13.1 \mathrm{~mm}$ on day 7 and $11.2 \mathrm{~mm}$ on day 14 . The persistent $D F$ in the $G-14$ increased from $12.6 \mathrm{~mm}$ on day 7 to $16.4 \mathrm{~mm}$ on day $14(P<0.001)$. The number of follicles $\geq 8 \mathrm{~mm}, 48 \mathrm{~h}$ after the beginning of the FSH treatment, was 15.6 in G-7 and 12.5 in G-14 $(P<0.05)$. No treatment effects were detected on number of corpus luteum and embryos. The shorter interval between follicular recruitment the higher the number of recruited follicles, but it did not have effects on the number or quality of embryos produced.
\end{abstract}

Keywords: dairy cow, follicular ablation, embryo, FSH, superovulation

Recebido em 11 de novembro de 2005

Aceito em 10 de abril de 2007

*Autor para correspondência (corresponding author)

E-mail: vasconcelos@fca.unesp.br 


\section{INTRODUÇÃO}

Em vacas, o desenvolvimento folicular durante o ciclo estral normal é caracterizado por duas a três ondas de crescimento (Sirois e Fortune, 1988; Ginther et al., 1989). Cada onda consiste na emergência simultânea de um grupo de folículos, denominada de recrutamento folicular, que é estimulado por aumento transitório do FSH (Turzilo e Fortune, 1990; Adams et al., 1992). Aproximadamente três dias após a emergência folicular, quando o maior folículo atinge o diâmetro de aproximadamente $8,5 \mathrm{~mm}$, um folículo continua crescendo e se torna o folículo dominante (FD), enquanto os demais folículos regridem e se tornam subordinados (FS), num evento denominado desvio ou seleção (Ginther et al., 1996).

Um dos objetivos da superovulação é fazer com que grande número de folículos atinja a condição ovulatória, para aumentar a produção de embriões. A resposta superovulatória tem grande variação, o que reduz a eficiência e a lucratividade dos programas de transferência de embriões. Estudos (Monniaux et al., 1983) relataram que a condição ovariana no início do tratamento superovulatório é responsável pela grande variabilidade da resposta superovulatória, tanto no número quanto na qualidade dos embriões produzidos.

Alguns dados sugerem que um pequeno intervalo entre os recrutamentos foliculares pode influenciar o recrutamento e/ou o desenvolvimento folicular subseqüente. A aplicação de três doses consecutivas de GnRH com intervalos de sete dias aumentou a porcentagem de dupla ovulação de $33 \%$, após a segunda dose de GnRH, para 47\%, após a terceira (Vasconcelos et al., 1999). A aspiração folicular, por sua vez, quando realizada duas vezes por semana, aumentou o número de folículos disponíveis e a porcentagem de recuperação dos ovócitos em relação à aspiração folicular feita uma única vez por semana (Garcia e Salaheddine, 1998).

O objetivo deste trabalho foi avaliar o efeito do intervalo entre os recrutamentos foliculares, de sete e 14 dias, na subseqüente resposta superovulatória, avaliada pelo número e pela qualidade dos embriões produzidos, em vacas da raça Holandesa não-lactantes.

\section{MATERIAL E MÉTODOS}

Vacas da raça Holandesa não-lactantes, com idade de quatro a 10 anos, foram utilizadas como doadoras de embrião $(n=13)$. Os animais foram mantidos em pasto e suplementados com canade-açúcar triturada, $2,5 \mathrm{~kg} / \mathrm{vaca} / \mathrm{dia}$ de farelo de trigo e sal mineral à vontade.

Utilizou-se sêmen de dois touros com características semelhantes: um deles foi usado para inseminar nove vacas e o outro quatro, em todos os períodos.

As vacas foram tratadas em quatro períodos. No período 1, elas foram distribuídas aleatoriamente em dois grupos, de acordo com o intervalo entre os recrutamentos foliculares: no grupo 7, o intervalo entre recrutamentos foliculares foi de sete dias e, no grupo 14, de 14 dias. Nos períodos subseqüentes elas receberam pelo menos uma vez cada tratamento, alternadamente, perfazendo um total de 40 colheitas de embriões.

No início de cada período, todas as vacas foram sincronizadas pelo protocolo Ovsynch modificado, que consistiu na aplicação de uma dose de GnRH em dia aleatório do ciclo estral, seguido, seis dias depois, de aplicação de duas doses de $\mathrm{PGF}_{2 \alpha}$, com intervalo de 12 horas entre aplicações; 48 horas após a primeira aplicação de $\mathrm{PGF}_{2 \alpha}$, foi aplicada a segunda dose de GnRH (Roy e Twagiramungu, 1996).

A diferenciação entre os grupos foi feita no sétimo dia do ciclo estral (dia $0=$ dia da segunda aplicação de GnRH do protocolo Ovsynch). No grupo 7 , os animais $(\mathrm{n}=19)$ receberam um dispositivo intravaginal de progesterona previamente utilizado por cinco dias em outras vacas, para redução da concentração de progesterona $\left(\mathrm{CIDR}^{1}\right)$, e $100 \mathrm{mcg}$ de $\mathrm{GnRH}^{2}$, para a ovulação do folículo dominante e recrutamento folicular. Os animais do grupo $14 \quad(n=21)$ receberam um dipositivo intravaginal de progesterona usado previamente por cinco dias e $25 \mathrm{mg}$ de $\mathrm{PGF}_{2 \alpha}{ }^{3}$, para a regressão do corpo lúteo (CL) e manutenção de níveis sublúteos de progesterona e conseqüente manutenção do FD (ausência de recrutamento folicular).

${ }^{1}$ CIDR $®$ Pfizer - Saúde Animal, Brasil

${ }^{2}$ Cystorelin ${ }^{\circledR}$, Merial Ltd., EUA

${ }^{3}$ Lutalyse ${ }^{\circledR}$, Pfizer - Saúde Animal, Brasil 
A partir do dia 14, os dois grupos receberam o mesmo tratamento superovulatório. Nesse dia, foi feita a aspiração do folículo dominante e dos folículos $\geq 4 \mathrm{~mm}$ e a troca do dispositivo intravaginal por outro, novo, associado à aplicação de $25 \mathrm{mg}$ de $\mathrm{PGF}_{2 \alpha}{ }^{3}$. O tratamento superovulatório começou 30 a 36 horas após a aspiração, com aplicação de $280 \mathrm{mg}$ de $\mathrm{FSH}^{4}$ em oito doses decrescentes, com intervalo de oito horas. O dispositivo de progesterona foi retirado no momento da aplicação da sétima dose de $\mathrm{FSH}^{4}$. Trinta e seis horas depois de retirado o dispositivo, as vacas receberam $100 \mathrm{mcg}$ de $\mathrm{GnRH}^{2}$ e foram inseminadas 12 e 24 horas depois.

Os exames ultra-sonográficos foram realizados com aparelho $\mathrm{Aloka}^{5}$, com transdutor linear retal de 7,5MHz; para a aspiração folicular, foi utilizado um transdutor vaginal convexo de $5,0 \mathrm{MHz}$, montado em armação de plástico contendo um guia para agulha de $47 \mathrm{~cm}$ de comprimento. A agulha utilizada tinha a medida 17 (diâmetro interno $=1,2 \mathrm{~mm}$; diâmetro externo $=1,5 \mathrm{~mm})$.

Foram avaliados por ultra-sonografia a ovulação ao final do protocolo "Ovsynch", a ovulação do FD no grupo 7 após a aplicação de $\mathrm{GnRH}^{2}$ no dia sete, a regressão do CL no grupo 14 após a aplicação de $\mathrm{PGF}_{2 \alpha}{ }^{3}$ no dia sete, a onda de crescimento folicular, o número de ondas foliculares e o intervalo entre os recrutamentos foliculares. Após a aspiração folicular, também foi avaliado se o FD tinha sido efetivamente removido, para sincronizar a emergência da nova onda de crescimento folicular.

Nos dois grupos, só foram superovuladas as vacas que ovularam ao segundo GnRH do protocolo inicial de sincronização (Ovsynch). No grupo 7 , só continuaram no tratamento as vacas que responderam à aplicação de GnRH no dia sete do tratamento e apresentaram novo recrutamento folicular. No grupo 14, só foram superovuladas as vacas que apresentaram luteólise após a aplicação de PGF $2 \alpha$ no dia sete, mantiveram o folículo dominante por 14 dias e não apresentaram outro recrutamento folicular no período.

${ }^{4}$ Folltropin-V®, Vetrepharm Inc., Canada

${ }^{5}$ Aloka - modelo SSD500-V, EUA
Foram colhidas amostras de sangue nos dias sete, 14 e 19 do protocolo para dosagem das concentrações séricas de estradiol e progesterona. As amostras de sangue, colhidas da veia coccígea em tubos com vácuo e sem anticoagulante, foram imediatamente colocadas no gelo em posição vertical após a colheita e mantidas a $4^{\circ} \mathrm{C}$ por 24 horas. Após centrifugação a $1200 \mathrm{~g}$ por 15 minutos e separação do soro, foram armazenadas a $-20{ }^{\circ} \mathrm{C}$ até a realização das dosagens.

As concentrações séricas de progesterona foram determinadas com kit de radioimunoensaio em fase sólida ${ }^{6}$, validado para o uso em vacas. As amostras foram processadas em dois ensaios com coeficiente de variação (CV) intra-ensaio para as amostras com concentração baixa e alta de 5,4\%, $7,9 \%$ e $0,3 \%$ e $2,1 \%$, respectivamente, e o $\mathrm{CV}$ interensaio para as mesmas concentrações de $1,9 \%$ e $0,9 \%$.

As concentrações séricas de estradiol foram determinadas com kit de radioimunoensaio ${ }^{7}$, validado para o uso em vacas. As amostras foram processadas em dois ensaios com CV intraensaio para as amostras com concentração baixa e alta de $3,0 \%, 26,9 \%$ e $20,7 \%, 12,1 \%$, respectivamente, e o $\mathrm{CV}$ interensaio para as mesmas concentrações de $14,0 \%$ e $15,5 \%$.

Os embriões foram recuperados sete dias depois da inseminação artificial, pela técnica de colheita não cirúrgica. Foram classificados quanto ao estádio de desenvolvimento e à qualidade, de acordo com os critérios da Sociedade Internacional de Transferência de Embriões. Para a análise final, os embriões foram classificados em viáveis (graus 1, 2 e 3), degenerados (graus 4 e 5) e não fertilizados. Após a classificação os embriões foram congelados.

Os resultados de número de folículos foram submetidos à transformação $\mathrm{y}^{\prime}=\sqrt{\mathrm{y}}$, e os demais dados relativos à contagem (número de corpos lúteos, número de embriões viáveis, degenerados e não fertilizados) à transformação $\mathrm{y}^{\prime}=\sqrt{\mathrm{y}+0,5}$.

${ }^{6}$ Coat a Count ${ }^{\circledR}$ - Diagnostic Products Corporation, EUA

${ }^{7} 3^{\text {rd }}$ Generation Estradiol RIA - Diagnostic Systems Laboratories, EUA

Arq. Bras. Med. Vet. Zootec., v.59, n.4, p.844-850, 2007 
A análise estatística das variáveis: concentração de progesterona, número de folículos, número de corpos lúteos, número de embriões viáveis, degenerados e não fertilizados foi feita por meio do programa MIXED do SAS (User's..., 1988). No modelo foram incluídos os efeitos fixos de tratamento, período e seqüência de tratamentos, bem como o efeito aleatório de vaca dentro de seqüência.

\section{RESULTADOS}

A taxa de sincronização ao protocolo inicial ("Ovsynch") foi de $100 \%$ (43/43). Foram consideradas sincronizadas as vacas que não apresentavam o FD 48 horas após a aplicação do segundo $\mathrm{GnRH}$ e apresentaram formação de CL correspondente ao folículo que ovulou. No grupo 7, recrutamento folicular a cada sete dias, a taxa de ovulação, em resposta à aplicação de GnRH no dia sete, foi de $95,2 \%(20 / 21)$. No grupo 14 , recrutamento folicular a cada 14 dias, a taxa de luteólise, em resposta à aplicação de prostaglandina no dia sete foi de 100\% (22/22); todas as vacas apresentaram regressão estrutural do CL, 48 horas após a aplicação de PGF2 $\alpha$. A taxa de manutenção do FD persistente no grupo 14 foi de $95,5 \%(21 / 22)$.

As diferenças na concentração de progesterona, no diâmetro do FD e na concentração de estradiol no dia 14 do experimento, confirmam a persistência do FD e a ausência de novo recrutamento folicular no grupo 14 e o recrutamento de uma nova onda de crescimento folicular no grupo 7, devido à ovulação em resposta à aplicação de GnRH no dia sete (Tab. 1).

Tabela 1. Diâmetro do folículo dominante (FD), concentrações séricas de progesterona e de estradiol nos dias sete, 14 e 19 do experimento, de acordo com intervalo entre os recrutamentos foliculares

\begin{tabular}{lccc}
\hline $\begin{array}{c}\text { Intervalo entre recrutamentos } \\
\text { foliculares }\end{array}$ & $\begin{array}{c}\text { Diâmetro do } \\
\text { FD }(\mathrm{mm})\end{array}$ & $\begin{array}{c}\text { Concentração de } \\
\text { progesterona }(\mathrm{ng} / \mathrm{ml})\end{array}$ & $\begin{array}{c}\text { Concentração de } \\
\text { estradiol (pg/ml) }\end{array}$ \\
\hline 7 dias (grupo 7) & & & \\
Dia 7 & $13,1 \pm 0,57$ & $2,0 \pm 0,21$ & $2,0 \pm 0,37$ \\
Dia 14 & $11,2 \pm 0,57^{* * *}$ & $5,9 \pm 0,44^{* *}$ & $1,1 \pm 0,37^{*}$ \\
Dia 19 & -- & $0,2 \pm 0,04$ & $7,6 \pm 1,78$ \\
14 dias (grupo 14) & & & \\
Dia 7 & $12,6 \pm 0,55$ & $1,9 \pm 0,21$ & $1,6 \pm 0,36$ \\
Dia 14 & $16,4 \pm 0,55^{* * *}$ & $1,2 \pm 0,43^{* *}$ & $2,5 \pm 0,36^{*}$ \\
Dia 19 & -- & $0,10 \pm 0,03$ & $6,9 \pm 1,76$ \\
\hline
\end{tabular}

Comparações entre os grupos 7 e 14 no dia 14 do tratamento: * $\mathrm{P}<0,01 ; * * \mathrm{P}>0,001 ; * * * \mathrm{P}<0,0001$.

O resultado a ser destacado é o número de folículos maiores ou iguais a $8 \mathrm{~mm}, 48$ horas após o início do tratamento com $\mathrm{FSH}$, que foi maior $(\mathrm{P}<0,05)$ no grupo 7 que no grupo 14, mostrando que o intervalo de sete dias entre os recrutamentos foliculares influenciou positivamente o número de folículos recrutados pelo tratamento superovulatório subseqüente (Tab. 2).
Apesar do maior número de folículos recrutados no grupo 7 , não foi encontrada diferença $(\mathrm{P}>0,10)$ no número de corpos lúteos e de embriões, entre os grupos 7 e 14, (Tab. 2). Também não foi observada diferença na taxa de recuperação dos embriões entre os grupos $7(55 \%)$ e $14(53 \%)$.

Não foi detectada diferença na qualidade dos embriões produzidos $(\mathrm{P}>0,10)$ entre os grupos $7 \mathrm{e}$ 14 (Tab. 3).

Tabela 2. Número de folículos, de corpos lúteos e de embriões, de acordo com o intervalo entre os recrutamentos foliculares

\begin{tabular}{lcccc}
\hline $\begin{array}{l}\text { Intervalo entre } \\
\text { recrutamentos foliculares }\end{array}$ & $\mathrm{N}$ & $\begin{array}{c}\text { Número de } \\
\text { folículos }\end{array}$ & Número de corpos lúteos & Número de embriões \\
\hline 7 dias (grupo 7) & 19 & $15,6 \pm 0,05 \mathrm{a}$ & $9,9 \pm 0,10$ & $5,4 \pm 0,07$ \\
14 dias (grupo 14) & 21 & $12,5 \pm 0,05 \mathrm{~b}$ & $9,8 \pm 0,10$ & $5,2 \pm 0,08$ \\
\hline
\end{tabular}

${ }^{\#}$ Foram considerados os folículos maiores ou iguais a $8 \mathrm{~mm}, 48$ horas após o início do tratamento com FSH. Valores seguidos por letras distintas na coluna deferem entre si $(\mathrm{P}<0,05)$. 
Tabela 3. Número de embriões viáveis, estruturas não fertilizadas e embriões degenerados, de acordo com o intervalo entre os recrutamentos foliculares

\begin{tabular}{lcccc}
\hline $\begin{array}{l}\text { Intervalo entre } \\
\text { recrutamentos } \\
\text { foliculares }\end{array}$ & $\mathrm{N}$ & $\begin{array}{c}\text { Embriões } \\
\text { viáveis }\end{array}$ & $\begin{array}{c}\text { Estruturas não } \\
\text { fertilizados }\end{array}$ & $\begin{array}{c}\text { Embriões } \\
\text { degenerados }^{2}\end{array}$ \\
\hline 7 dias (grupo 7) & 19 & $3,0 \pm 0,08$ & $0,9 \pm 0,03$ & $1,5 \pm 0,11$ \\
14 dias (grupo 14) & 21 & $2,6 \pm 0,08$ & $1,1 \pm 0,03$ & $1,6 \pm 0,11$ \\
\hline
\end{tabular}

${ }^{\mathrm{T}}$ Embriões classificados como graus 1,2 e $3 ;{ }^{2}$ Embriões classificados como graus 4 e 5.

\section{DISCUSSÃO}

Os resultados indicam que o intervalo de 14 dias entre os recrutamentos foliculares (grupo 14) reduziu o número de folículos recrutados pelo tratamento superovulatório subseqüente. $\mathrm{O}$ menor número de folículos recrutados no grupo $14(12,5 \pm 0,05)$ comparado com o grupo 7 $(15,6 \pm 0,05)$ pode ser explicado pela ação do FD sobre secreção de FSH pela glândula pituitária (Gibbons et al., 1997; Gibbons et al., 1999; Bleach et al., 2001). Estudos in vivo (Fortune et al., 1998) e in vitro (Lussier et al., 1994) demonstraram que folículos primários de bovinos não dependem de FSH para se desenvolverem, porém o decréscimo na concentração de FSH, causado pela ação do FD, pode precipitar a atresia dos folículos de 3 a $4 \mathrm{~mm}$ de diâmetro, quando estes começam a expressar dependência ao FSH (Adams et al., 1992; Turzillo e Fortune , 1993).

No grupo 7, como o recrutamento foi a cada sete dias, o FD exerceu sua ação sobre os demais folículos por um período menor, possibilitando que maior número de folículos estivessem aptos para serem recrutados pela ação do FSH exógeno do tratamento superovulatório, enquanto, no grupo 14 , recrutamento a cada 14 dias, folículos de 3 a $4 \mathrm{~mm}$ de diâmetro ficaram sob a ação do FD por um período maior, provavelmente aumentando o número de folículos que entraram em atresia, pois já expressavam dependência ao FSH.

Apesar do maior recrutamento folicular no grupo 7, não foi detectada diferença no número de CL entre os grupos. O número de corpos lúteos dividido pelo número de folículos (48 horas após o início do tratamento com FSH) indica que a taxa de ovulação pode ter sido menor no grupo 7 $(63 \%)$ que no grupo 14 (79\%). Segundo D’Occhio et al. (1999), as falhas de ovulação em protocolos de superovulação podem ser devido ao curto período de desenvolvimento dos folículos durante o tratamento superovulatório. Estudos (Sartori et al. (2001) mostraram que os folículos adquirem capacidade ovulatória com aproximadamente $10 \mathrm{~mm}$ de diâmetro (um dia após o desvio), mas precisam de maior concentração de LH para ovularem do que folículos maiores. A aquisição da capacidade ovulatória provavelmente envolve o aumento da expressão de receptores de LH nas células da granulosa. No grupo 7, devido ao maior número de folículos recrutados $(15,6 \pm 0,05$ vs. $12,5 \pm 0,05$ folículos), o estímulo gonadotrófico talvez não tenha sido suficiente para o completo desenvolvimento de todos os folículos e/ou aquisição da capacidade ovulatória, reduzindo, assim, a taxa de ovulação.

Não foi encontrada diferença na concentração de estradiol no dia 19 (Tab. 1), entre os grupos 7 $(7,6 \pm 1,78 \mathrm{pg} / \mathrm{ml})$ e $14(6,9 \pm 1,76 \mathrm{pg} / \mathrm{ml})$. Esse dado, associado ao número de $\mathrm{CL}$, sugere que o desenvolvimento dos folículos recrutados no grupo 7 pode ter sido incompleto. $\mathrm{O}$ esperado seria maior concentração de estradiol no dia 19 (final do tratamento superovulatório) e maior número de CL nos animais do grupo 7, se todos os folículos recrutados tivessem chegado ao desenvolvimento final e adquirido capacidade ovulatória.

A qualidade dos embriões não foi influenciada pelo intervalo entre os recrutamentos foliculares, sendo que os dois grupos apresentaram a mesma proporção de embriões viáveis, degenerados e não fertilizados (Tab. 3). Provavelmente porque, após a aspiração folicular, o tratamento superovulatório foi igual para os grupos 7 e 14, e foi usado um dispositivo intravaginal de progesterona na ausência de CL (aplicação de $\mathrm{PGF}_{2 \alpha}$ no dia da aspiração folicular), para que a concentração de progesterona fosse uniforme 
entre os grupos, pois, sabe-se que a progesterona poderia influenciar a pulsatilidade de LH, interferindo no desenvolvimento final dos folículos (Stock e Fortune, 1993; Bergfeld et al., 1996;) e na qualidade dos ovócitos (Revah e Butler, 1996).

Outro fator que poderia influenciar a qualidade dos embriões é a concentração de estradiol. Altas concentrações de estradiol na circulação periférica de vacas, devido ao crescimento de grande número de folículos, podem ser prejudiciais à fertilização e/ou ao desenvolvimento embrionário (Gong et al., 1993). Adicionalmente, Binelli et al. (1999) mostraram que a secreção de proteínas do oviducto é alterada por altas concentrações de estradiol. No presente estudo, apesar de o recrutamento folicular ter sido maior no grupo 7 , não foi encontrada diferença na concentração de estradiol no dia 19 (final do tratamento superovulatório; Tab. 1), provavelmente porque o mesmo número de folículos chegou ao desenvolvimento final nos dois grupos, o que pode ser estimado pelo número de CL, que foi igual entre os grupos (Tab. 2). Portanto, não houve influência da concentração de estradiol sobre a qualidade dos embriões em nenhum dos grupos.

Estes resultados não suportam a hipótese inicial de que o menor intervalo entre os recrutamentos foliculares melhoraria a resposta ao tratamento superovulatório subseqüente.

O tratamento foi eficiente para sincronizar o estro no final da superovulação. Das 40 vacas superovuladas, $37(92,5 \%)$ apresentaram sinais de estro até $36 \mathrm{~h}$ após a retirada do dispositivo intravaginal de progesterona, que ocorreu junto à sétima aplicação de FSH. Destas, 21,6\% (8/37) iniciaram o estro 24 horas após a remoção do dispositivo intravaginal de progesterona, e 78,4\% (29/37) 36 horas após a remoção do dispositivo, no momento da aplicação do GnRH. Essa alta taxa de sincronização ao final do protocolo de superovulação se deve à aspiração folicular 36 horas antes do início do tratamento superovulatório. Foi verificado (Gibbons et al., 1999) que a emergência da nova onda ocorre aproximadamente 30 horas após a aspiração do FD e foi relatado (Bergfelt et al., 1994) alto grau de sincronização da emergência da nova onda após a aspiração de todos os folículos e também alta taxa de sincronização da ovulação quando administraram prostaglandina quatro dias após a aspiração folicular. Estes resultados indicam a viabilidade da utilização da inseminação artificial com tempo fixo em protocolos de superovulação.

\section{CONCLUSÃO}

Em vacas da raça Holandesa não-lactantes, o menor intervalo entre os recrutamentos foliculares (sete vs. 14 dias) aumentou o número de folículos recrutados pelo tratamento superovulatório com FSH subseqüente, porém não teve efeito sobre a quantidade e a qualidade dos embriões produzidos.

\section{REFERÊNCIAS BIBLIOGRÁFICAS}

ADAMS, G.P.; MATTERI, R.L.; KO, J.C.H. et al. Association between surges of folliclestimulating hormone and emergence of follicular waves in heifers. J. Reprod. Fertil., v.94, p.177188, 1992.

BERGFELD, E.G.M.; KOJIMA, F.N.; CUPP, A.S. et al. Changing dose of progesterona results in sudden changes in frequency of luteinizing hormone pulses and secretion of $17 \beta$-estradiol in bovine females. Biol. Reprod., v.54, p.546-553, 1996.

BERGFELT, D.R.; LIGHTFOOT, K.C.; ADAMS, G.P. Ovarian synchronization following ultrasound - guide transvaginal follicle ablation in heifers. Theriogenology, v.42, p.895907, 1994.

BINELLI, M.; HAMPTON, J.; BUHI, W.C. et al. Persistent dominant follicle alters pattern of oviductal secretory proteins from cows at estrus. Biol. Reprod., v.61, p.127-134, 1999.

BLEACH, E.C.L.; GLENCROSS, R.G.; FEIST, S.A. et al. Plasma inhibin $A$ in heifers: Relationship with follicle dynamics, gonadotropins, and steroids during the estrous cycle and after treatment with bovine follicular fluid. Biol. Reprod., v.64, p.743-752, 2001.

D’OCCHIO, M.J.; JILLELLA, D.; LINDSEY, B.R. Factors that influence follicle recruitment, growth and ovulation during ovarian superstimulation in heifers: Opportunities to 
increase ovulation rate and embryo recovery by delaying the exposure of follicles to $\mathrm{LH}$. Theriogenology, v.51, p.9-35, 1999.

FORTUNE, J.E.; KITO, S.; WANDJI, S.A. et al. Activation of bovine and baboon primordial follicles in vitro. Theriogenology, v.49, p.441449, 1998.

GARCIA, A.; SALAHEDDINE, M. Effects of repeated ultrasound-guide transvaginal follicular aspiration on bovine oocyte recovery and subsequent follicular development. Theriogenology, v.50, p.575-585, 1998.

GIBBONS, J.R.; WILTBANK, M.C.; GINTHER, O.J. Functional interrelationships between follicles greater than $4 \mathrm{~mm}$ and the follicle-stimulating hormone surge in heifers. Biol. Reprod., v.57, p.1066-1073, 1997.

GIBBONS, J.R.; WILTBANK, M.C.; GINTHER, O.J. Relationship between follicular development and the decline in the folliclestimulating hormone surge in heifers. Biol. Reprod., v.60, p.72-77, 1999.

GINTHER, O.J.; KASTELIC, J.P.; KNOPF, L. Composition and characteristics of follicular waves during the bovine estrus cycle. Anim. Reprod. Sci., v.20, p.187-200, 1989.

GINTHER, O.J.; WILTBANK, M.C.; FRICKE, P.M. et al. Selection of the Dominant Follicle in Cattle. Biol. Reprod., v.55, p.1187-1194, 1996.

GONG, J.G.; TONY, A.; BRAMILEY, A. et al. Effect of recombinant bovine somatotropin on the superovulatory responses to pregnant mare serum gonadotropin in heifers. Biol. Reprod., v.48, p.1141-1149, 1993.

LUSSIER, J.G.; MATTON, P.; GUILBAULT, L.A. et al. Ovarian follicular development and endocrine responses in follicular-fluid-treated and hemi-ovariectomized heifers. J. Reprod. Fertil., v.102, p.95-105, 1994.
MONNIAUX, D.; CHUPIN, D.; SAUMANDE, J. Superovulatory responses in cattle. Theriogenology, v.19, p.55-81, 1983.

REVAH, I.; BUTLER, W.R. Prolonged dominance of follicles and reduced viability of bovine oocytes. J. Reprod. Fertil., v.106, p.3947, 1996.

ROY, G.L.; TWAGIRAMUNGU, H. A fixed time IA program using the GnRH - PGF GnRH method for beef females. J. Anim. Sci., v.74, p.462, 1996. (Abstr.).

SARTORI, R.; FRICKE, P.M.; FERREIRA, J.C.P. et al. Follicular deviation and acquisition of ovulatory capacity in bovine follicles. Biol. Reprod., v.65, p.1403-1409, 2001.

SIROIS, J.; FORTUNE, J.E. Ovarian follicular dynamics during the estrous cycle in heifers monitored by real-time ultrasonography. Biol. Reprod., v.39, p.308-317, 1988.

STOCK, A. E.; FORTUNE, J.E. Ovarian follicular dominance in cattle: relationship between prolonged growth of the ovulatory follicle and endocrine parameters. Endocrinology, v.132, p.1108-1114, 1993.

TURZILLO, A.M.; FORTUNE, J.E. Effects of suppressing plasma FSH on ovarian follicular dominance in cattle. J. Reprod. Fertil., v.98, p.113-119, 1993.

TURZILLO, A.M.; FORTUNE, J.E. Suppression of the secondary FSH surge with bovine follicular fluid is associated with delayed ovarian follicular development in heifers. J. Reprod. Fertil., v.89, p.643-653, 1990.

USER'S guide: Statistics, Version 6.02. Cary, NC: SAS Institute, 1988.

VASCONCELOS, J.L.M.; SANTOS, R.M.; COSTA, D.A. et al. Effects of progesterona concentration on size of the ovulatory follicle and double ovulation rate in lactating dairy cows. J. Dairy Sci., v.82, p.99, 1999. (Abstr.). 